\title{
Pressure-Induced Phase Transitions in Danburite-Type
}

\section{Borosilicates}

Liudmila A. Gorelova ${ }^{1 *}$, Anna S. Pakhomova², Maria G. Krzhizhanovskayal', B. Winkler ${ }^{3}$, Sergey V. Krivovichev ${ }^{1,4}$, Leonid S. Dubrovinsky

${ }^{1}$ Department of Crystallography, Institute of Earth Sciences, St. Petersburg State University, University Emb. 7/9, 199034 St. Petersburg, Russia

2 Deutsches Elektronen-Synchrotron (DESY), Petra III, Notkestraße 85, 22607 Hamburg,

Germany

${ }^{3}$ Institute für Geowissenschaften, Frankfurt University, Altenhöferallee 1, DE-60438 Frankfurt am Main, Germany

${ }^{4}$ Kola Science Centre, Russian Academy of Sciences, Fersman str. 14, 184209 Apatity,

Russia

${ }^{5}$ Bayerisches Geoinstitut, University of Bayreuth, Universitätsstraße 30, 95447 Bayreuth,

Germany 
Table S1. Crystallographic data and refinement parameters for maleevite $\mathrm{BaB}_{2} \mathrm{Si}_{2} \mathrm{O}_{8}$

\begin{tabular}{|c|c|c|c|c|c|c|c|c|c|c|c|c|c|}
\hline $\begin{array}{l}\text { Pressure point } \\
\text { Pressure, GPa }\end{array}$ & $\begin{array}{l}\text { P0 } \\
0.0001\end{array}$ & $\begin{array}{l}\text { P01 } \\
4.21(1)\end{array}$ & $\begin{array}{l}\mathrm{P} 02 \\
7.96(1)\end{array}$ & $\begin{array}{l}\text { P03 } \\
11.63(1)\end{array}$ & $\begin{array}{l}\text { P04 } \\
13.94(1)\end{array}$ & $\begin{array}{l}\text { P05 } \\
17.53(1)\end{array}$ & $\begin{array}{l}\text { P06 } \\
19.69(1)\end{array}$ & $\begin{array}{l}\text { P07 } \\
22.35(1)\end{array}$ & $\begin{array}{l}\text { P08 } \\
24.98(1)\end{array}$ & $\begin{array}{l}\text { P09 } \\
28.65(1)\end{array}$ & $\begin{array}{l}\text { P10 } \\
33.22(1)\end{array}$ & $\begin{array}{l}\text { P11 } \\
35.61(1)\end{array}$ & $\begin{array}{l}\text { P12 } \\
38.30(1)\end{array}$ \\
\hline Phase name & & \multicolumn{11}{|c|}{ maleevite-I } & maleevite-II \\
\hline Space group & \multicolumn{12}{|c|}{ Pnma } & $P-1$ \\
\hline$a, \AA / \alpha,{ }^{\circ}$ & $8.1528(7)$ & $7.7480(2)$ & $7.4060(2)$ & $7.2018(2)$ & $7.1335(7)$ & $7.062(1)$ & $6.9996(13)$ & $6.983(3)$ & $6.926(2)$ & $6.883(2)$ & $6.793(6)$ & $6.755(4)$ & $5.630(4) / 92.07(6)$ \\
\hline$b, \AA / \beta,^{\circ}$ & $8.1917(5)$ & $8.1850(2)$ & $8.1975(1)$ & $8.1867(1)$ & $8.1533(3)$ & $8.1096(6)$ & $8.0984(6)$ & $8.056(1)$ & $8.038(1)$ & $8.0038(9)$ & $8.007(3)$ & $8.021(2)$ & $5.538(4) / 103.97(7)$ \\
\hline$c, \AA / \gamma,{ }^{\circ}$ & $9.0484(2)$ & $8.8893(2)$ & $8.7543(2)$ & $8.6403(2)$ & $8.5755(4)$ & $8.5043(9)$ & $8.4488(8)$ & $8.389(2)$ & $8.346(2)$ & $8.307(2)$ & $8.211(5)$ & $8.182(3)$ & $6.746(6) / 94.98(6)$ \\
\hline Volume, $\AA^{3}$ & $604.30(7)$ & $563.74(2)$ & $531.48(2)$ & $509.42(2)$ & $498.76(6)$ & $487.0(1)$ & $478.9(1)$ & $471.9(2)$ & $464.6(2)$ & $457.6(2)$ & $446.6(5)$ & $443.3(3)$ & $203.0(3)$ \\
\hline$Z$ & & & & & & & 4 & & & & & & 1 \\
\hline \multicolumn{14}{|l|}{ Data collection } \\
\hline Wavelength, $\AA$ & 0.2891 & & & & & & 0.41106 & & & & & & 0.41106 \\
\hline Max. $\theta^{\circ}$ & 17.976 & 20.917 & 20.857 & 20.809 & 20.729 & 20.617 & 20.717 & 20.669 & 20.626 & 20.678 & 20.221 & 20.617 & 14.123 \\
\hline Index ranges & $-10 \leq h \leq 12$ & $-10 \leq h \leq 8$ & $-7 \leq h \leq 9$ & $-7 \leq h \leq 9$ & $-9 \leq h \leq 6$ & $-9 \leq h \leq 7$ & $-9 \leq h \leq 7$ & $-9 \leq h \leq 7$ & $-7 \leq h \leq 9$ & $-6 \leq h \leq 9$ & $-6 \leq h \leq 8$ & $-6 \leq h \leq 8$ & $-6 \leq h \leq 6$ \\
\hline & $-13 \leq k \leq 14$ & $-10 \leq k \leq 10$ & $-10 \leq k \leq 10$ & $-10 \leq k \leq 10$ & $-10 \leq k \leq 10$ & $-9 \leq k \leq 9$ & $-9 \leq k \leq 9$ & $-9 \leq k \leq 9$ & $-9 \leq k \leq 9$ & $-9 \leq k \leq 9$ & $-9 \leq k \leq 9$ & $-9 \leq k \leq 9$ & $-6 \leq k \leq 6$ \\
\hline & $-18 \leq l \leq 19$ & $-12 \leq l \leq 10$ & $-12 \leq l \leq 10$ & $-11 \leq l \leq 12$ & $-12 \leq l \leq 10$ & $-12 \leq l \leq 10$ & $-12 \leq l \leq 10$ & $-10 \leq l \leq 12$ & $-12 \leq l \leq 10$ & $-12 \leq l \leq 9$ & $-11 \leq l \leq 10$ & $-11 \leq l \leq 9$ & $-5 \leq l \leq 6$ \\
\hline No.meas.refl. & 2283 & 1593 & 1324 & 1364 & 1233 & 1196 & 1216 & 1199 & 1116 & 1064 & 574 & 1020 & 328 \\
\hline No.uniq.refl. & 1040 & 737 & 658 & 649 & 617 & 585 & 587 & 585 & 580 & 549 & 264 & 491 & 208 \\
\hline $\begin{array}{l}\text { No. obs.refl } \\
(I>2 \sigma(I))\end{array}$ & 986 & 682 & 556 & 535 & 470 & 490 & 460 & 421 & 489 & 452 & 160 & 357 & 206 \\
\hline \multicolumn{14}{|c|}{ Refinement of the structure } \\
\hline No.of variables & 65 & 64 & 64 & 64 & 54 & 64 & 64 & 64 & 64 & 64 & 64 & 64 & 58 \\
\hline$R_{\text {int }}$ & 0.0262 & 0.0225 & 0.0260 & 0.0363 & 0.0397 & 0.0290 & 0.0409 & 0.0476 & 0.0417 & 0.0320 & 0.0696 & 0.0453 & 0.0210 \\
\hline$R_{1}$, all data & 0.0222 & 0.0270 & 0.0511 & 0.0497 & 0.0917 & 0.0640 & 0.0802 & 0.0762 & 0.0588 & 0.0855 & 0.1105 & 0.1073 & 0.1066 \\
\hline$R_{1}, I>2 \sigma(I)$ & 0.0209 & 0.0249 & 0.0413 & 0.0364 & 0.0744 & 0.0557 & 0.0681 & 0.0559 & 0.0492 & 0.0773 & 0.0645 & 0.0859 & 0.1060 \\
\hline $\mathrm{w} R_{2}$, all data & 0.0560 & 0.0690 & 0.1016 & 0.1159 & 0.2134 & 0.1643 & 0.2029 & 0.1733 & 0.1400 & 0.2282 & 0.2046 & 0.2645 & 0.2651 \\
\hline $\mathrm{w} R_{2}, I>2 \sigma(I)$ & 0.0516 & 0.0628 & 0.0914 & 0.0979 & 0.1924 & 0.1533 & 0.1861 & 0.1482 & 0.1266 & 0.2165 & 0.1632 & 0.2320 & 0.2633 \\
\hline GooF & 1.118 & 1.102 & 1.123 & 1.163 & 1.196 & 1.128 & 1.116 & 1.100 & 1.089 & 1.141 & 1.090 & 1.098 & 1.496 \\
\hline
\end{tabular}


Table S2. Crystallographic data and refinement parameters for pekovite $\mathrm{SrB}_{2} \mathrm{Si}_{2} \mathrm{O}_{8}$

\begin{tabular}{|c|c|c|c|c|c|c|c|c|c|c|}
\hline $\begin{array}{l}\text { Pressure point } \\
\text { Pressure, GPa }\end{array}$ & $\begin{array}{l}\mathrm{P} 0 \\
0.0001 \\
\end{array}$ & $\begin{array}{l}\text { P01 } \\
1.61(1) \\
\end{array}$ & $\begin{array}{l}\mathrm{P} 02 \\
5.87(1) \\
\end{array}$ & $\begin{array}{l}\text { P03 } \\
9.32(1) \\
\end{array}$ & $\begin{array}{l}\text { P04 } \\
14.20(1) \\
\end{array}$ & $\begin{array}{l}\text { P05 } \\
18.06(1) \\
\end{array}$ & $\begin{array}{l}\text { P06 } \\
23.06(1) \\
\end{array}$ & $\begin{array}{l}\text { P07 } \\
25.85(1) \\
\end{array}$ & $\begin{array}{l}\text { P08 } \\
29.42(1) \\
\end{array}$ & $\begin{array}{l}\text { P09 } \\
33.28(1)\end{array}$ \\
\hline Phase name & \multicolumn{6}{|c|}{ pekovite-I } & \multicolumn{3}{|c|}{ pekovite-II } & pekovite-III \\
\hline Space group & \multicolumn{6}{|c|}{ Pnma } & \multicolumn{3}{|c|}{ Pnma } & $P-1$ \\
\hline$a, \AA / \alpha,{ }^{\circ}$ & $8.1457(5)$ & $8.0419(8)$ & $7.6002(5)$ & $7.3298(6)$ & $7.0809(8)$ & $6.9539(5)$ & $6.8235(14)$ & $6.7771(13)$ & $6.6968(6)$ & $5.43(2) / 104.6(4)$ \\
\hline$b, \AA / \beta, \circ$ & $7.9238(3)$ & $7.9221(4)$ & $7.9743(3)$ & $7.9849(3)$ & $7.9675(4)$ & $7.9516(2)$ & $7.8974(5)$ & $7.8812(5)$ & $7.8686(4)$ & $5.51(2) / 96.0(4)$ \\
\hline$c, \AA / \gamma,{ }^{\circ}$ & $8.9224(3)$ & $8.8796(4)$ & $8.6902(2)$ & $8.5681(3)$ & $8.4312(3)$ & $8.3498(2)$ & $8.2609(5)$ & $8.2158(5)$ & $8.1593(3)$ & $6.55(3) / 95.4(3)$ \\
\hline Volume, $\AA^{3}$ & $575.90(4)$ & $565.71(7)$ & $526.68(4)$ & $501.46(5)$ & $475.66(6)$ & $461.70(4)$ & $445.16(10)$ & $438.82(9)$ & $429.95(5)$ & $187(1)$ \\
\hline$Z$ & \multicolumn{6}{|c|}{$4^{500.00(4)}$} & \multicolumn{3}{|c|}{4} & 1 \\
\hline \multicolumn{11}{|l|}{ Data collection } \\
\hline Wavelength, $\AA$ & \multicolumn{6}{|c|}{0.2894} & \multicolumn{3}{|c|}{0.2894} & 0.2894 \\
\hline Max. $\theta^{\circ}$ & 18.315 & 17.984 & 18.190 & 18.331 & 18.249 & 18.374 & 18.220 & 18.302 & 18.178 & 14.193 \\
\hline Index ranges & $-12 \leq h \leq 13$ & $-11 \leq h \leq 10$ & $-9 \leq h \leq 10$ & $-8 \leq h \leq 9$ & $-9 \leq h \leq 8$ & $-8 \leq h \leq 8$ & $-8 \leq h \leq 8$ & $-9 \leq h \leq 8$ & $-8 \leq h \leq 8$ & $-10 \leq h \leq 11$ \\
\hline & $-13 \leq k \leq 13$ & $-13 \leq k \leq 12$ & $-12 \leq k \leq 13$ & $-12 \leq k \leq 13$ & $-13 \leq k \leq 12$ & $-12 \leq k \leq 13$ & $-12 \leq k \leq 13$ & $-12 \leq k \leq 13$ & $-12 \leq k \leq 13$ & $-10 \leq k \leq 11$ \\
\hline & $-18 \leq l \leq 16$ & $-15 \leq l \leq 13$ & $-14 \leq l \leq 13$ & $-14 \leq l \leq 13$ & $-14 \leq l \leq 13$ & $-14 \leq l \leq 13$ & $-13 \leq l \leq 14$ & $-13 \leq l \leq 14$ & $-13 \leq l \leq 14$ & $-9 \leq l \leq 8$ \\
\hline No.meas.refl. & 2579 & 3181 & 2783 & 2654 & 2448 & 2441 & 2337 & 2453 & 2327 & 31 \\
\hline No.uniq.refl. & 1258 & 1245 & 1090 & 1030 & 975 & 961 & 917 & 954 & 905 & \\
\hline $\begin{array}{l}\text { No. obs.refl } \\
(I>2 \sigma(I))\end{array}$ & 1168 & 969 & 964 & 924 & 861 & 846 & 740 & 719 & 778 & \\
\hline \multicolumn{11}{|c|}{ Refinement of the structure } \\
\hline No.of variables & 65 & 31 & 52 & 64 & 64 & 65 & 73 & 73 & 73 & \multirow{7}{*}{0.4218} \\
\hline$R_{\text {int }}$ & 0.0341 & 0.0569 & 0.0468 & 0.0465 & 0.0435 & 0.0403 & 0.0377 & 0.0562 & 0.0245 & \\
\hline$R_{1}$, all data & 0.0301 & 0.2161 & 0.0989 & 0.0485 & 0.0437 & 0.0830 & 0.0609 & 0.0880 & 0.0474 & \\
\hline$R_{1}, I>2 \sigma(I)$ & 0.0282 & 0.1304 & 0.0720 & 0.0322 & 0.0316 & 0.0595 & 0.0447 & 0.0568 & 0.0399 & \\
\hline $\mathrm{w} R_{2}$, all data & 0.0798 & 0.4917 & 0.2722 & 0.0907 & 0.0878 & 0.2627 & 0.1496 & 0.2202 & 0.1100 & \\
\hline $\mathrm{w} R_{2}, I>2 \sigma(I)$ & 0.0773 & 0.3098 & 0.1826 & 0.0825 & 0.0818 & 0.1776 & 0.1342 & 0.1692 & 0.1012 & \\
\hline GooF & 1.110 & 2.211 & 1.231 & 1.065 & 1.064 & 1.184 & 1.131 & 1.138 & 1.086 & \\
\hline
\end{tabular}


Table S3. Experimental pressure points for Raman study of maleevite and pekovite

\begin{tabular}{llll}
\hline \multicolumn{2}{c}{ Compression } & \multicolumn{2}{c}{ Decompression } \\
\hline Pressure point & Pressure, GPa & Pressure point & Pressure, GPa \\
\hline P00 & 0.0001 & & \\
P01 & $0.56(1)$ & P01d & $36.16(1)$ \\
P02 & $1.64(1)$ & P02d & $32.86(1)$ \\
P03 & $3.29(1)$ & P03d & $30.34(1)$ \\
P04 & $4.51(1)$ & P04d & $26.11(1)$ \\
P05 & $5.86(1)$ & P05d & $21.16(1)$ \\
P06 & $7.30(1)$ & P06d & $16.62(1)$ \\
P07 & $7.97(1)$ & P07d & $11.89(1)$ \\
P08 & $9.46(1)$ & P08d & $7.57(1)$ \\
P09 & $10.96(1)$ & P09d & $3.93(1)$ \\
P10 & $12.47(1)$ & & \\
P11 & $13.79(1)$ & & \\
P12 & $15.21(1)$ & & \\
P13 & $16.75(1)$ & & \\
P14 & $17.97(1)$ & & \\
P15 & $19.40(1)$ & & \\
P16 & $20.51(1)$ & & \\
P17 & $21.89(1)$ & & \\
P18 & $23.13(1)$ & & \\
P19 & $24.33(1)$ & & \\
P20 & $25.83(1)$ & & \\
P21 & $27.26(1)$ & & \\
P22 & $28.66(1)$ & & \\
P23 & $30.41(1)$ & & \\
P24 & $31.70(1)$ & & \\
P25 & $32.62(1)$ & & \\
P26 & $34.43(1)$ & & \\
P27 & $35.93(1)$ & & \\
P28 & $37.81(1)$ & & \\
P29 & $39.68(1)$ & & \\
\hline
\end{tabular}


Table S4. Pressure-induced changes of maleevite $\mathrm{BaB}_{2} \mathrm{Si}_{2} \mathrm{O}_{8}$, pekovite $\mathrm{SrB}_{2} \mathrm{Si}_{2} \mathrm{O}_{8}$ and danburite $\mathrm{CaB}_{2} \mathrm{Si}_{2} \mathrm{O}_{8}$. Calculations for danburite at room pressure were performed using the atomic coordinates from Sugiyama \& Takeuchi ${ }^{24}$ and Pakhomova et al. ${ }^{20}$ for high pressures.

\begin{tabular}{|c|c|c|c|c|c|c|c|c|c|c|c|c|c|}
\hline \multicolumn{14}{|c|}{ Maleevite } \\
\hline Pressure, $\mathrm{GPa}$ & 0.0001 & $4.21(1)$ & $7.96(1)$ & $11.63(1)$ & 13.94(1) & $17.53(1)$ & $19.69(1)$ & $22.35(1)$ & $24.98(1)$ & $28.65(1)$ & $33.22(1)$ & $35.61(1)$ & $\Delta_{0.0001-35.61}$ \\
\hline \multicolumn{14}{|c|}{ Deformations of 8-membered ring } \\
\hline$L(\mathrm{O} 5-\mathrm{O} 5), \AA$ & 8.627 & 8.534 & 8.504 & 8.496 & 8.49 & 8.451 & 8.405 & 8.402 & 8.377 & 8.363 & 8.33 & 8.29 & 0.340 \\
\hline$S(\mathrm{O} 5-\mathrm{O} 5), \AA$ & 3.605 & 3.324 & 3.039 & 2.840 & 2.76 & 2.700 & 2.668 & 2.622 & 2.581 & 2.542 & 2.44 & 2.45 & 1.137 \\
\hline$L / S$ & 2.39 & 2.57 & 2.80 & 2.99 & 3.08 & 3.13 & 3.15 & 3.20 & 3.25 & 3.29 & 3.41 & 3.38 & \\
\hline \multicolumn{14}{|c|}{ Deformations of 4-membered ring } \\
\hline $\mathrm{O} 1-\mathrm{O} 2-\mathrm{O} 1, \stackrel{\circ}{ }$ & 98.34 & 103.93 & 106.5 & 107.2 & 107.7 & 107.9 & 107.9 & 109.1 & 109.0 & 109.7 & 110.4 & 112.1 & -14.03 \\
\hline $\mathrm{O} 2-\mathrm{O} 1-\mathrm{O} 2,^{\circ}$ & 81.66 & 76.07 & 73.5 & 72.8 & 72.3 & 72.1 & 72.1 & 70.9 & 71.0 & 70.3 & 69.6 & 67.9 & 14.53 \\
\hline $\mathrm{O} 1-\mathrm{O} 1, \AA$ & 3.861 & 3.990 & 4.018 & 4.003 & 4.02 & 4.017 & 4.00 & 4.02 & 3.990 & 4.02 & 3.97 & 4.06 & -0.215 \\
\hline $\mathrm{O} 2-\mathrm{O} 2, \AA$ & 3.338 & 3.124 & 3.005 & 2.954 & 2.943 & 2.928 & 2.912 & 2.86 & 2.847 & 2.84 & 2.76 & 2.73 & 0.610 \\
\hline \multicolumn{14}{|c|}{ Contraction of $\mathrm{Ba}-\mathrm{Ba}$ distances } \\
\hline $\mathrm{Ba}-\mathrm{Ba}, \AA$ & 4.728 & 4.871 & 5.063 & 5.184 & 5.214 & 5.247 & 5.265 & 5.274 & 5.279 & 5.284 & 5.508 & 5.331 & -0.610 \\
\hline $\mathrm{Ba}-\mathrm{Ba}^{\prime}, \AA$ & 6.503 & 6.289 & 6.115 & 6.013 & 5.964 & 5.909 & 5.879 & 5.838 & 5.813 & 5.783 & 5.734 & 5.737 & 0.761 \\
\hline $\mathrm{Ba}-\mathrm{Ba} ", \AA$ & 5.080 & 4.828 & 4.630 & 4.505 & 4.454 & 4.396 & 4.350 & 4.321 & 4.280 & 4.242 & 4.167 & 4.136 & 0.926 \\
\hline
\end{tabular}

\begin{tabular}{|c|c|c|c|c|c|c|c|c|c|c|}
\hline \multicolumn{11}{|c|}{ Pekovite } \\
\hline Pressure, GPa & 0.0001 & $1.61(1)$ & $5.87(1)$ & $9.32(1)$ & $14.20(1)$ & $18.06(1)$ & $23.06(1)$ & $25.85(1)$ & $29.42(1)$ & $\Delta_{0.0001-29.42}$ \\
\hline \multicolumn{11}{|c|}{ Deformations of 8-membered ring } \\
\hline$L(\mathrm{O} 4-\mathrm{O} 4), \AA$ & 8.772 & 8.750 & 8.625 & 8.544 & 8.455 & 8.396 & 8.306 & 8.273 & 8.219 & 0.553 \\
\hline$S(\mathrm{O} 5-\mathrm{O} 5), \AA$ & 3.380 & 3.303 & 3.031 & 2.860 & 2.704 & 2.631 & 2.600 & 2.580 & 2.540 & 0.840 \\
\hline$L / S$ & 2.60 & 2.65 & 2.85 & 2.99 & 3.13 & 3.19 & 3.19 & 3.21 & 3.24 & \\
\hline \multicolumn{11}{|c|}{ Deformations of 4-membered ring } \\
\hline $\mathrm{O} 1-\mathrm{O} 2-\mathrm{O} 1,{ }^{\circ}$ & 92.93 & 91.90 & 81.32 & 77.84 & 75.70 & 74.3 & 73.49 & 72.83 & 72.22 & 20.71 \\
\hline $\mathrm{O} 2-\mathrm{O} 1-\mathrm{O} 2,^{\circ}$ & 87.07 & 88.10 & 98.68 & 102.16 & 104.30 & 105.7 & 106.51 & 107.17 & 107.78 & -20.71 \\
\hline $\mathrm{O} 1-\mathrm{O} 1, \AA$ & 3.690 & 3.654 & 3.282 & 3.145 & 3.044 & 2.972 & 2.969 & 2.933 & 2.907 & 0.783 \\
\hline $\mathrm{O} 2-\mathrm{O} 2, \AA$ & 3.507 & 3.535 & 3.819 & 3.892 & 3.915 & 3.922 & 3.973 & 3.972 & 3.982 & -0.475 \\
\hline \multicolumn{11}{|c|}{ Contraction of $\mathrm{Sr}-\mathrm{Sr}$ distances } \\
\hline $\mathrm{Sr}-\mathrm{Sr}, \AA$ & 4.586 & 4.626 & 4.862 & 5.009 & 5.143 & 5.206 & 5.412 & 5.408 & 5.399 & -0.813 \\
\hline $\mathrm{Sr}-\mathrm{Sr}, \AA$ & 6.371 & 6.314 & 6.093 & 5.963 & 5.847 & 5.792 & 5.715 & 5.693 & 5.668 & 0.703 \\
\hline $\mathrm{Sr}-\mathrm{Sr} ", \AA$ & 4.586 & 5.018 & 4.682 & 4.515 & 4.369 & 4.288 & 4.206 & 4.168 & 4.109 & 0.477 \\
\hline
\end{tabular}

Danburite

\begin{tabular}{|c|c|c|c|c|c|c|c|c|c|c|c|}
\hline Pressure, GPa & 0.0001 & $1.13(1)$ & $3.87(1)$ & $6.50(1)$ & $9.60(1)$ & $12.52(1)$ & $15.64(1)$ & $18.89(1)$ & $22.06(1)$ & $22.56(1)$ & $\Delta_{0.0001-22.56}$ \\
\hline \multicolumn{12}{|c|}{ Deformations of 8-membered ring } \\
\hline$L(\mathrm{O} 5-\mathrm{O} 5), \AA$ & 8.795 & 8.791 & 8.757 & 8.724 & 8.663 & 8.568 & 8.485 & 8.418 & 8.349 & 8.333 & 0.462 \\
\hline$S(\mathrm{O} 5-\mathrm{O} 5), \AA$ & 3.187 & 3.158 & 3.077 & 3.006 & 2.902 & 2.770 & 2.686 & 2.613 & 2.552 & 2.540 & 0.647 \\
\hline
\end{tabular}




\begin{tabular}{|c|c|c|c|c|c|c|c|c|c|c|c|}
\hline$L / S$ & 2.760 & 2.783 & 2.846 & 2.902 & 2.985 & 3.093 & 3.160 & 3.221 & 3.271 & 3.281 & \\
\hline \multicolumn{12}{|c|}{ Deformations of 4-membered ring } \\
\hline $\mathrm{O} 1-\mathrm{O} 2-\mathrm{O} 1,{ }^{\circ}$ & 81.79 & 81.72 & 81.64 & 82.22 & 86.21 & 95.57 & 100.04 & 102.53 & 104.57 & 105.15 & -23.36 \\
\hline $\mathrm{O} 2-\mathrm{O} 1-\mathrm{O} 2,{ }^{\circ}$ & 98.21 & 98.28 & 98.36 & 97.78 & 93.79 & 84.43 & 79.96 & 77.47 & 75.43 & 74.85 & 23.36 \\
\hline $\mathrm{O} 1-\mathrm{O} 1, \AA$ & 3.324 & 3.320 & 3.300 & 3.306 & 3.422 & 3.696 & 3.807 & 3.856 & 3.893 & 3.907 & -0.583 \\
\hline $\mathrm{O} 2-\mathrm{O} 2, \AA$ & 3.835 & 3.836 & 3.818 & 3.787 & 3.655 & 3.354 & 3.194 & 3.095 & 3.012 & 2.992 & 0.843 \\
\hline \multicolumn{12}{|c|}{ Contraction of $\mathrm{Ca}-\mathrm{Ca}$ distances } \\
\hline $\mathrm{Ca}-\mathrm{Ca}, \AA$ & 4.486 & 4.487 & 4.500 & 4.531 & 4.636 & 4.801 & 4.897 & 4.976 & 5.056 & 5.072 & -0.586 \\
\hline $\mathrm{Ca}-\mathrm{Ca}, \AA$ & 6.231 & 6.209 & 6.136 & 6.070 & 5.972 & 5.861 & 5.781 & 5.717 & 5.666 & 5.655 & 0.576 \\
\hline $\mathrm{Ca}-\mathrm{Ca},, \AA$ & 5.037 & 5.019 & 4.950 & 4.878 & 4.734 & 4.517 & 4.391 & 4.297 & 4.212 & 4.194 & 0.843 \\
\hline
\end{tabular}


Table S5. Bond distances and polyhedral parameters in maleevite-I, $\mathrm{BaB}_{2} \mathrm{Si}_{2} \mathrm{O}_{8}$

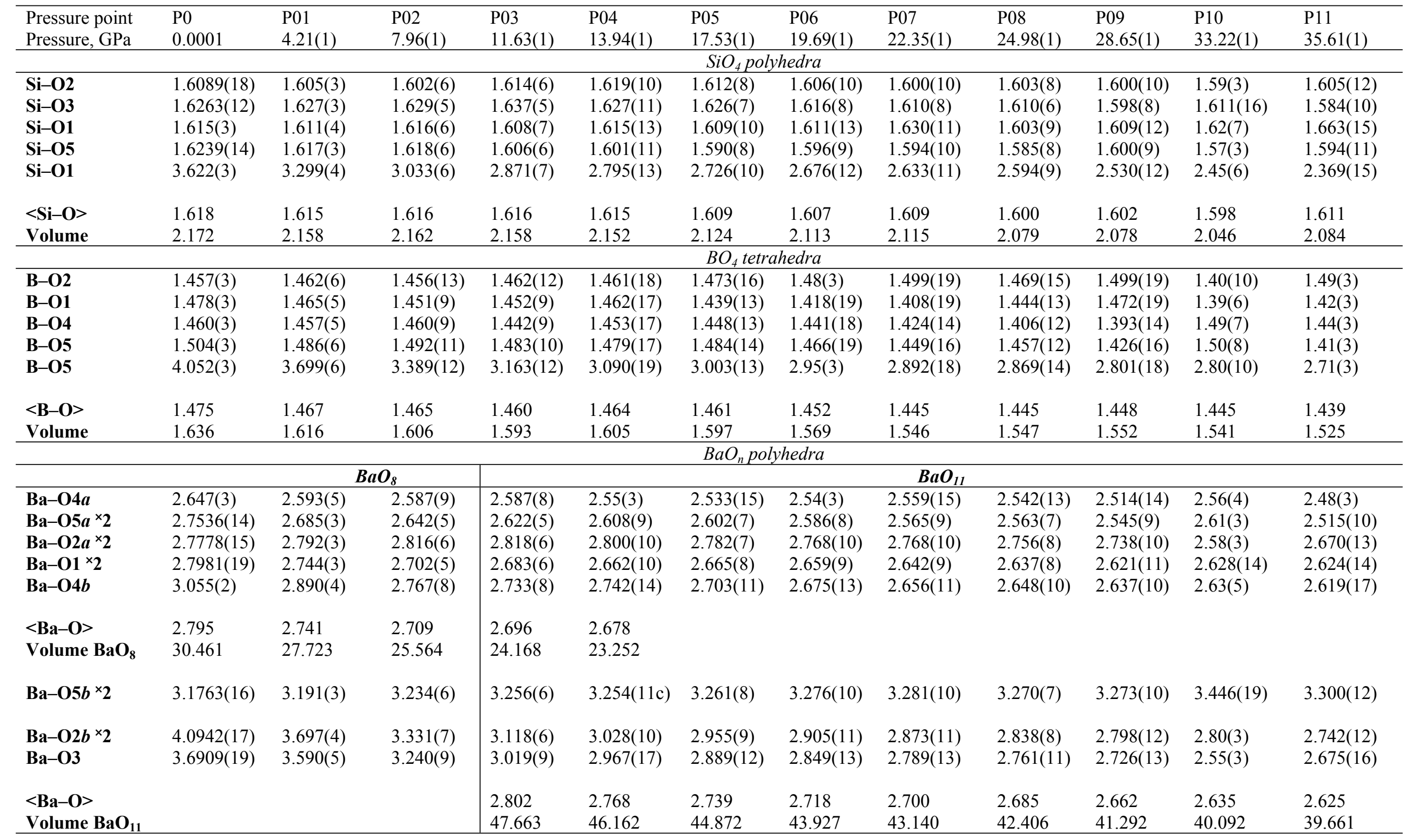


Table S6. Bond distances and polyhedral parameters in maleevite-II $\mathrm{BaB}_{2} \mathrm{Si}_{2} \mathrm{O}_{8}$ at $36.30(1) \mathrm{GPa}$

\begin{tabular}{|c|c|c|c|}
\hline \multicolumn{4}{|c|}{$\mathrm{SiO}_{6}$ polyhedra } \\
\hline Si1-04a & $1.84(10)$ & Si2-O7a & $1.93(9)$ \\
\hline $\mathbf{S i 1}-\mathbf{O} b$ & $1.72(4)$ & $\mathrm{Si} 2-\mathrm{O} 2$ & $1.70(6)$ \\
\hline Si1-O2 & $2.08(18)$ & $\mathrm{Si2}-04$ & $2.01(10)$ \\
\hline Si1-O8 & $1.93(7)$ & $\mathrm{Si} 2-06$ & $1.76(4)$ \\
\hline Si1-01 & $1.55(4)$ & Si2-08 & $1.72(8)$ \\
\hline Si1-O3 & $1.65(9)$ & $\mathrm{Si} 2-\mathrm{O} 7 b$ & $1.31(10)$ \\
\hline$<$ Si1-O $>$ & 1.79 & $<\mathbf{S i 2}-\mathbf{O}>$ & 1.74 \\
\hline Volume & 7.47 & Volume & 6.77 \\
\hline \multicolumn{4}{|c|}{$\mathrm{BO}_{4}$ polyhedra } \\
\hline B1-O3 & $1.8(3)$ & B2-O2 & $1.64(12)$ \\
\hline B1-07 & $1.53(13)$ & B2-O5 & $1.29(9)$ \\
\hline B1-O5 & $1.44(15)$ & B2-06 & $1.35(10)$ \\
\hline B1-08 & $1.68(12)$ & B2-O1 & $2.14(14)$ \\
\hline$<$ B1-O $>$ & 1.62 & $<\mathrm{B} 2-\mathrm{O}>$ & 1.61 \\
\hline Volume & 2.06 & Volume & 1.93 \\
\hline \multicolumn{4}{|c|}{$\mathrm{BaO}_{13}$ polyhedra } \\
\hline Ba-06a & $2.37(8)$ & $\mathrm{Ba}-05 a$ & $2.42(9)$ \\
\hline $\mathrm{Ba}-\mathrm{O} 2 a$ & $2.71(9)$ & Ba-O7 & $2.74(5)$ \\
\hline $\mathrm{Ba}-\mathrm{O} 2 \boldsymbol{b}$ & $3.11(18)$ & $\mathrm{Ba}-05 b$ & $2.50(7)$ \\
\hline $\mathrm{Ba}-\mathrm{O} 6 \boldsymbol{b}$ & $2.60(6)$ & Ba-O1b & $2.90(7)$ \\
\hline Ba-O4 & $2.51(8)$ & Ba-O3b & $2.56(7)$ \\
\hline Ba-O1a & $2.40(9)$ & Ba-08 & $2.50(5)$ \\
\hline $\mathrm{Ba}-\mathrm{O3a}$ & $2.62(6)$ & & \\
\hline$<\mathbf{B a}-\mathbf{O}>$ & 2.61 & & \\
\hline Volume $\mathrm{BaO}_{13}$ & 42.97 & & \\
\hline
\end{tabular}


Table S7. Bond distances and polyhedral parameters in pekovite $\mathrm{SrB}_{2} \mathrm{Si}_{2} \mathrm{O}_{8}$

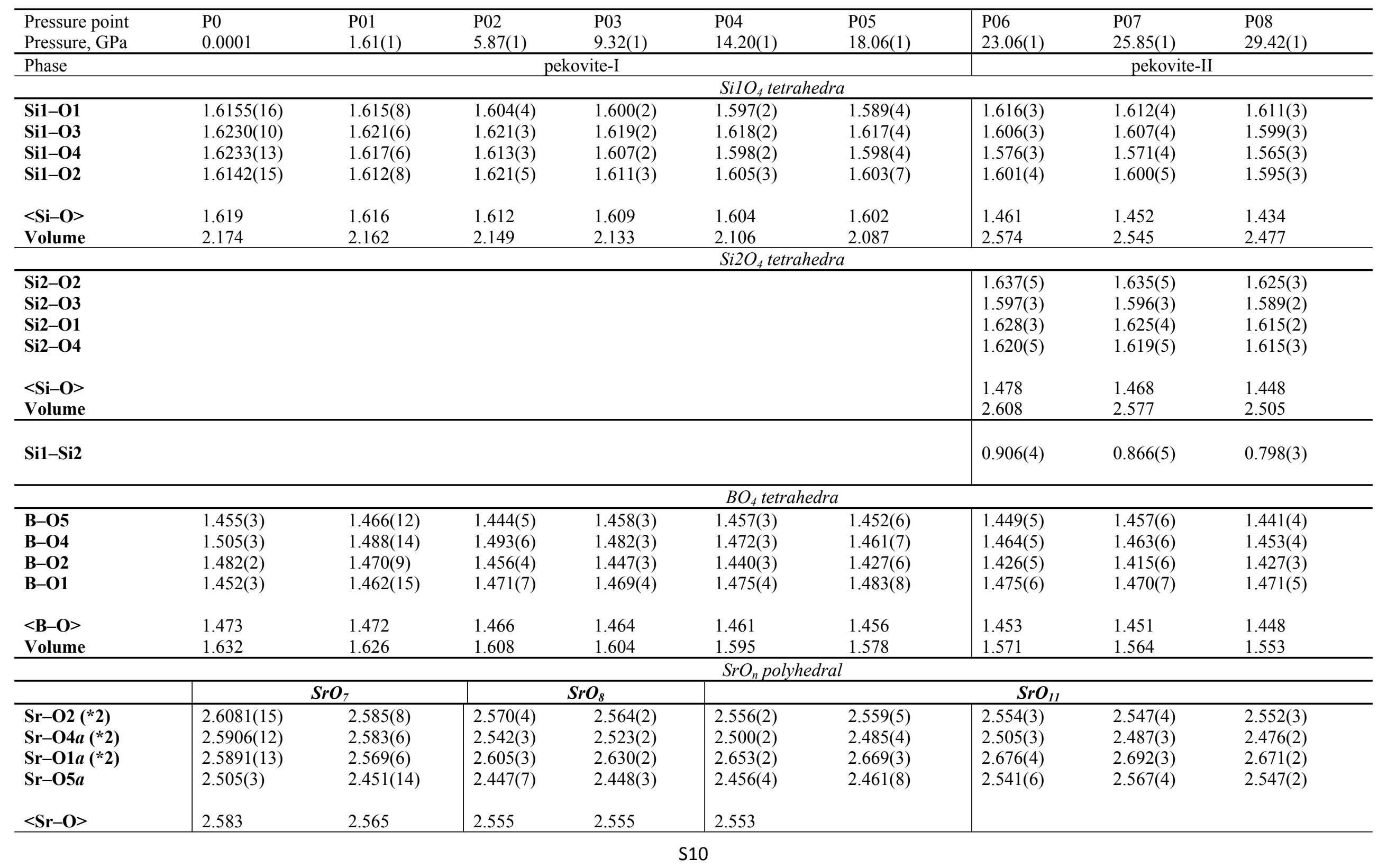




\begin{tabular}{|c|c|c|c|c|c|c|c|c|c|}
\hline $\begin{array}{l}\text { Volume } \mathrm{SrO}_{7} \\
\mathrm{Sr}-\mathrm{O5b}\end{array}$ & 21.422 & 20.711 & $\begin{array}{l}18.073 \\
2.938(6)\end{array}$ & $\begin{array}{l}16.633 \\
2.803(3)\end{array}$ & $\begin{array}{l}15.288 \\
2.707(3)\end{array}$ & $2.661(5)$ & $2.581(3)$ & $2.522(7)$ & $2.496(4)$ \\
\hline $\begin{array}{l}<\mathrm{Sr}-\mathrm{O}> \\
\text { Volume } \mathrm{SrO}_{8}\end{array}$ & & & $\begin{array}{l}2.602 \\
24.059\end{array}$ & $\begin{array}{l}2.586 \\
22.533\end{array}$ & $\begin{array}{l}2.573 \\
21.055\end{array}$ & $\begin{array}{l}2.568 \\
20.351\end{array}$ & & & \\
\hline $\begin{array}{l}\mathrm{Sr}-\mathrm{O} 4 b(* 2) \\
\mathrm{Sr}-\mathrm{O3}\end{array}$ & $3.0850(14)$ & $3.079(7)$ & & & $2.849(3)$ & $2.736(6)$ & $2.559(4)$ & $2.487(3)$ & $2.505(3)$ \\
\hline $\begin{array}{l}<\mathrm{Sr}-\mathrm{O}> \\
\text { Volume } \mathrm{SrO}_{9}\end{array}$ & $\begin{array}{l}2.694 \\
31.339\end{array}$ & $\begin{array}{l}2.679 \\
30.370\end{array}$ & & & $\begin{array}{l}2.603 \\
31.348\end{array}$ & $\begin{array}{l}2.587 \\
30.384\end{array}$ & & & \\
\hline $\mathrm{Sr}-\mathrm{O} 1 b(* 2)$ & & & & & 3.034 & $2.899(5)$ & $2.697(3)$ & $2.639(5)$ & $2.594(3)$ \\
\hline $\begin{array}{l}<\mathrm{Sr}-\mathrm{O}> \\
\text { Volume } \mathrm{SrO}_{11}\end{array}$ & & & & & $\begin{array}{l}2.683 \\
42.056 \\
\end{array}$ & $\begin{array}{l}2.644 \\
40.454 \\
\end{array}$ & $\begin{array}{l}2.595 \\
38.655 \\
\end{array}$ & $\begin{array}{l}2.577 \\
37.826 \\
\end{array}$ & $\begin{array}{l}2.558 \\
36.909 \\
\end{array}$ \\
\hline
\end{tabular}


Table S8. The orthogonalization coefficients in the crystal structures of danburite-group minerals $M \mathrm{~B}_{2} \mathrm{Si}_{2} \mathrm{O}_{8}(M=\mathrm{Ca}, \mathrm{Sr}$, $\mathrm{Ba})$.

\begin{tabular}{|c|c|c|c|c|c|c|}
\hline Pressure, GPa & $\chi_{1} ", \circ$ & $\chi_{1}^{\prime},{ }^{\circ}$ & $\Delta_{1}$ & $\chi_{2} ", \circ$ & $\chi_{2}^{\prime},{ }^{\circ}$ & $\Delta_{2}$ \\
\hline \multicolumn{7}{|c|}{ Maleevite } \\
\hline 0.0001 & 94.1 & 83.0 & 11.1 & 113.4 & 97.0 & 16.3 \\
\hline $4.21(1)$ & 89.2 & 83.3 & 6.0 & 113.3 & 96.8 & 16.6 \\
\hline $7.96(1)$ & 84.8 & 84.1 & 0.7 & 112.9 & 95.9 & 17.0 \\
\hline $11.63(1)$ & 82.3 & 84.1 & -1.8 & 112.1 & 95.9 & 16.2 \\
\hline $13.94(1)$ & 82.1 & 84.2 & -2.1 & 111.6 & 95.8 & 15.8 \\
\hline $17.53(1)$ & 80.7 & 84.2 & -3.5 & 111.1 & 95.8 & 15.3 \\
\hline $19.69(1)$ & 80.0 & 84.1 & -4.1 & 110.5 & 95.6 & 14.9 \\
\hline $22.35(1)$ & 80.0 & 83.4 & -3.4 & 109.9 & 96.6 & 13.3 \\
\hline $24.98(1)$ & 79.8 & 83.6 & -3.8 & 109.7 & 96.4 & 13.3 \\
\hline $28.65(1)$ & 79.4 & 82.6 & -3.2 & 109.2 & 97.4 & 11.8 \\
\hline $33.22(1)$ & 78.5 & 87.0 & -8.5 & 108.0 & 93.0 & 15.0 \\
\hline $35.61(1)$ & 78.1 & 85.0 & -6.9 & 107.6 & 95.0 & 12.6 \\
\hline \multicolumn{7}{|c|}{ Pekovite } \\
\hline 0.0001 & 96.1 & 81.1 & 15.0 & 110.2 & 98.9 & 11.3 \\
\hline $1.61(1)$ & 95.0 & 81.2 & 13.8 & 110.0 & 98.8 & 11.2 \\
\hline $5.87(1)$ & 88.5 & 81.6 & 6.8 & 110.4 & 98.4 & 12.0 \\
\hline $9.32(1)$ & 85.4 & 82.4 & 3.1 & 110.2 & 97.6 & 12.6 \\
\hline $14.21(1)$ & 82.6 & 83.0 & -0.4 & 109.8 & 97.0 & 12.8 \\
\hline $18.06(1)$ & 81.0 & 83.3 & -2.3 & 109.5 & 96.7 & 12.8 \\
\hline $23.06(1)^{*}$ & 78.7 & 98.7 & -19.9 & 114.2 & 81.4 & 32.8 \\
\hline $25.85(1)^{*}$ & 78.4 & 99.1 & -20.7 & 113.7 & 80.9 & 32.8 \\
\hline $29.42(1)^{*}$ & 77.7 & 100.1 & -22.4 & 113.2 & 79.9 & 33.3 \\
\hline \multicolumn{7}{|c|}{ Danburite $^{* *}$} \\
\hline $1.13(1)$ & 96.2 & 79.5 & 16.7 & 107.9 & 100.5 & 7.4 \\
\hline $3.87(1)$ & 95.2 & 79.4 & 15.8 & 107.5 & 100.6 & 6.9 \\
\hline $6.50(1)$ & 94.0 & 79.4 & 14.6 & 107.2 & 100.6 & 6.6 \\
\hline $9.60(1)$ & 91.3 & 79.6 & 11.7 & 107.3 & 100.4 & 6.9 \\
\hline $12.52(1)$ & 87.6 & 80.3 & 7.3 & 108.0 & 99.7 & 8.2 \\
\hline $15.64(1)$ & 85.3 & 80.7 & 4.6 & 108.1 & 99.3 & 8.8 \\
\hline $18.89(1)$ & 83.6 & 81.3 & 2.3 & 108.0 & 98.7 & 9.3 \\
\hline $22.06(1)$ & 81.9 & 82.1 & -0.3 & 107.8 & 97.9 & 9.9 \\
\hline $22.56(1)$ & 81.5 & 82.4 & -0.9 & 107.7 & 97.6 & 10.1 \\
\hline $25.36(1)$ & 73.8 & 91.4 & -17.6 & 101.4 & 88.6 & 12.8 \\
\hline
\end{tabular}

* for this pressures $\chi$ angles were calculated using $\mathrm{Si} 2$ atoms

** Calculations for danburite were performed using the atomic coordinates from Pakhomova et al. ${ }^{20}$. 

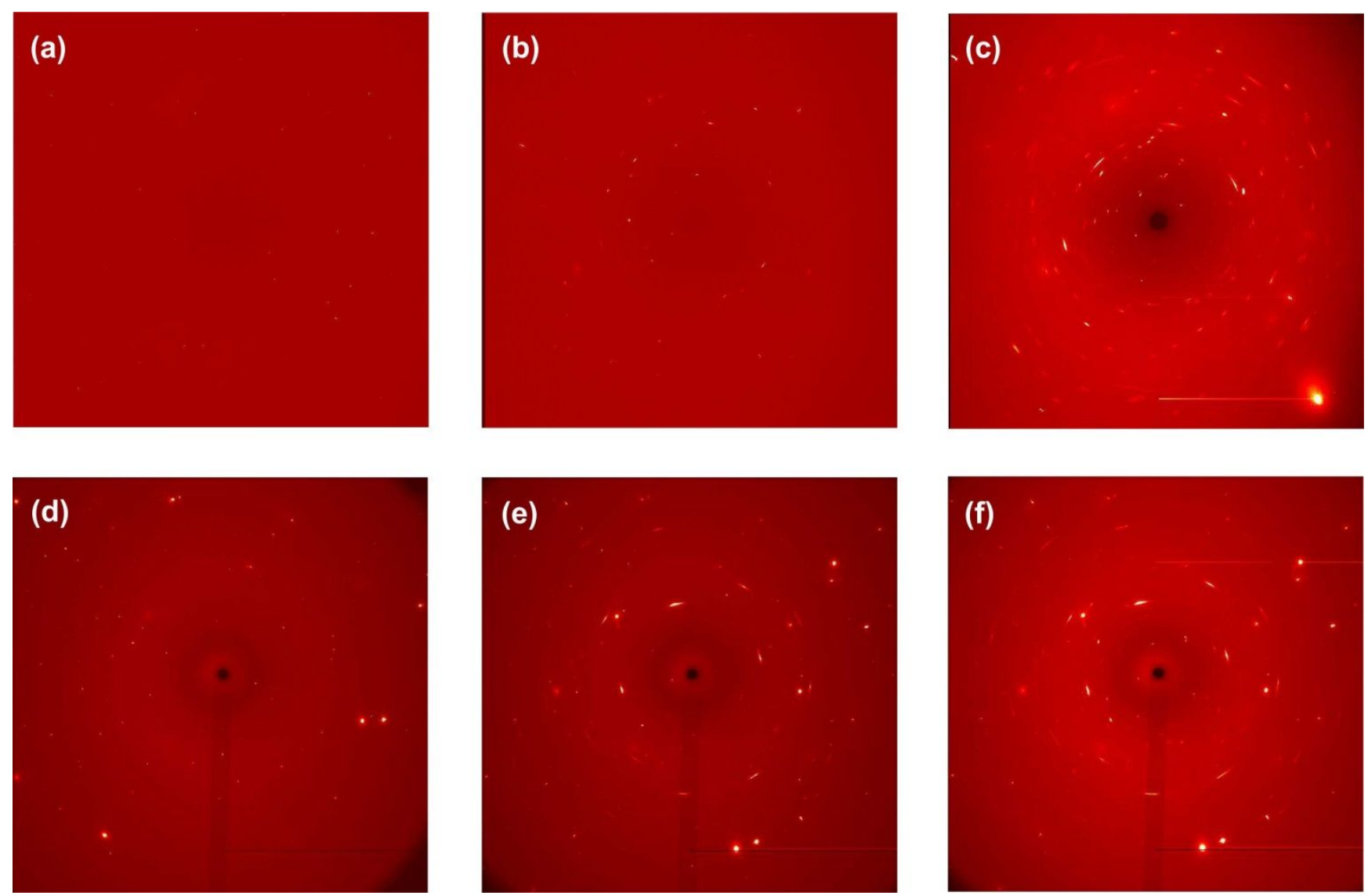

Figure S1. 2D X-ray diffraction data for maleevite-I at 4.2 GPa (a), 35.6 GPa (b), maleevite-II at 38.3 $\mathrm{GPa}(\mathrm{c})$ and for pekovite-I at 1.61 GPa (d), pekovite-II at 29.42 GPa (e), pekovite-III at 33.28 GPa (f). 

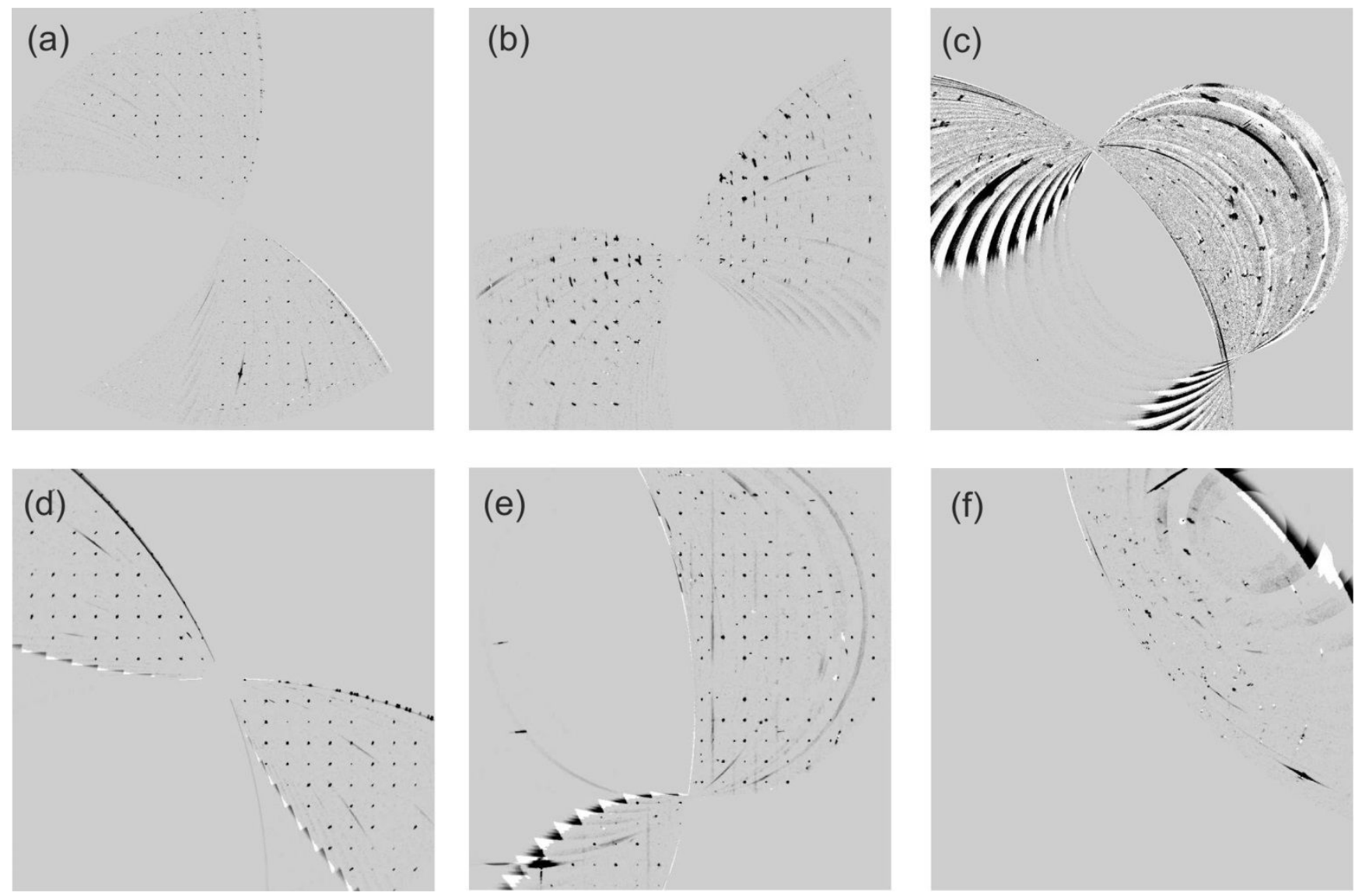

Figure S2. Reciprocal space reconstructions for maleevite (a,b,c) and pekovite (d,e,f) in the $h k-1$ plane. Maleevite-I at 4.2 GPa (a), 35.6 GPa (b), maleevite-II at 38.3 GPa (c) and pekovite-I at 1.61 $\mathrm{GPa}(\mathrm{d})$, pekovite-II at $29.42 \mathrm{GPa}(\mathrm{e})$, pekovite-III at $33.28 \mathrm{GPa}(\mathrm{f})$. 
(a)

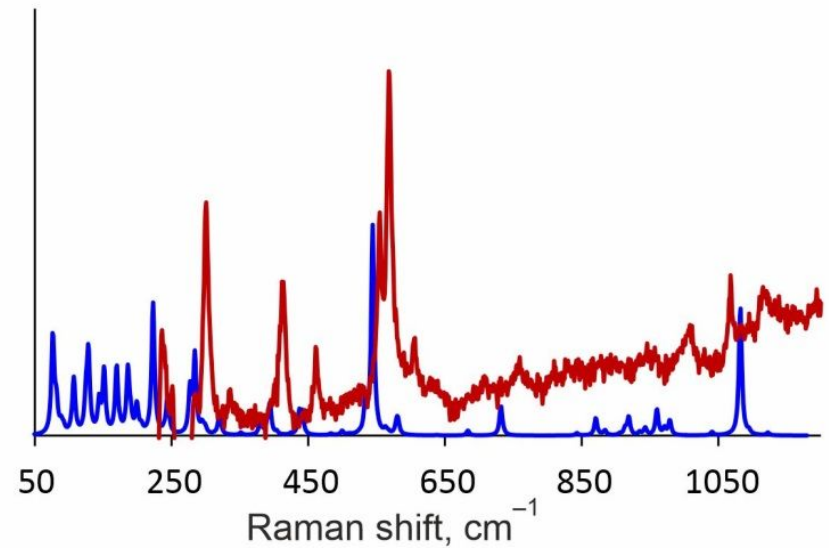

(b)

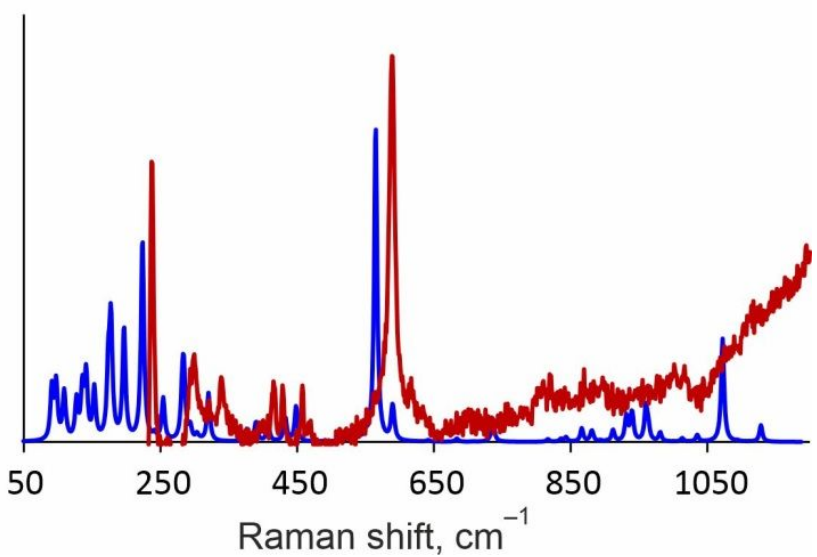

Figure S3. Comparison of an ambient pressure Raman spectrum of a natural maleevite (a) and pekovite (b) (red) samples with DFPT-based calculations (blue). 
(a)

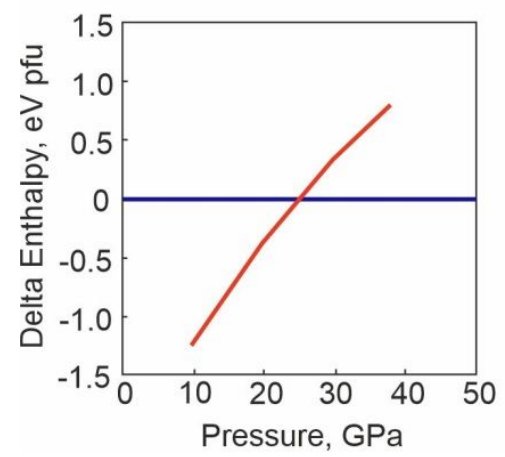

(b)

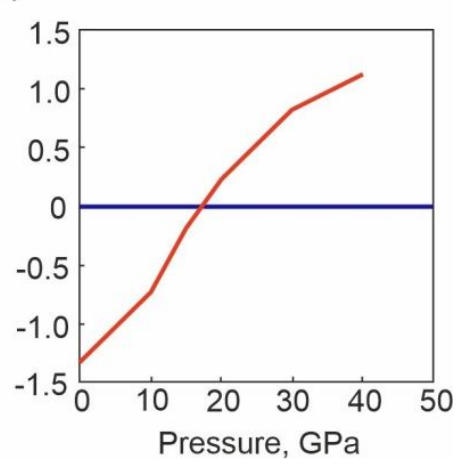

Figure S4. Pressure-dependence of the enthalpy difference between initial orthorhombic crystal structure and a high-pressure polymorph with the triclinic danburite-III structure type for maleevite $\mathrm{BaB}_{2} \mathrm{Si}_{2} \mathrm{O}_{8}$ (a) and pekovite $\mathrm{SrB}_{2} \mathrm{Si}_{2} \mathrm{O}_{8}$ (b). The phase transition, based on DFT-GGA calculations, occurs at about 25 and $17 \mathrm{GPa}$ for maleevite and pekovite, respectively. 
(a)

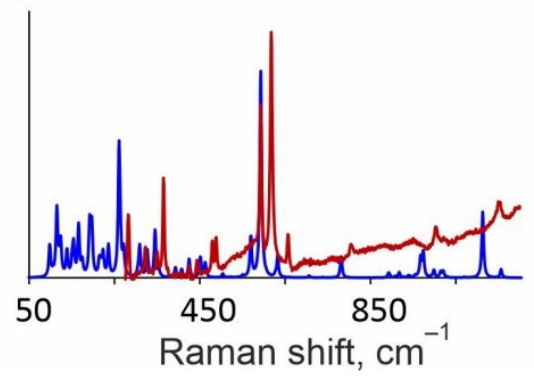

(b)

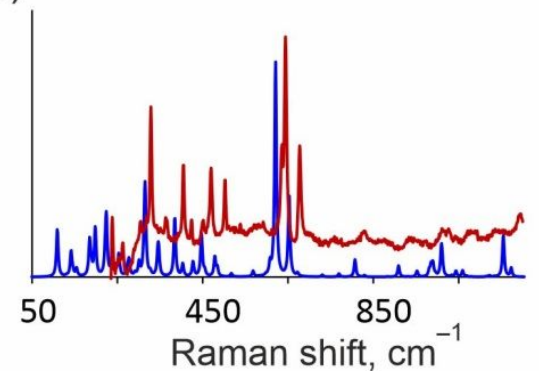

(c)

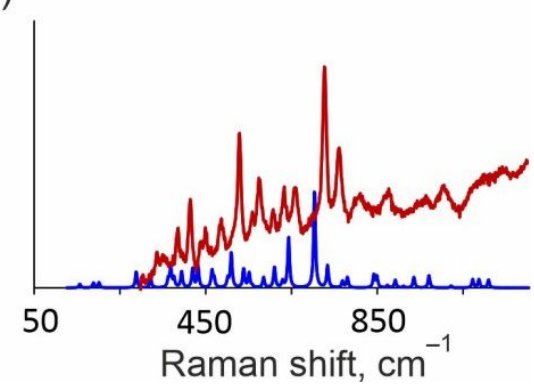

Figure S5. Comparison of the experimentally determined Raman spectrum of pekovite at different pressures (red) to results of DFPT calculations (blue): $8 \mathrm{GPa}$ (a), $20 \mathrm{GPa}$ (b) and $40 \mathrm{GPa}$ (c). 
(a)

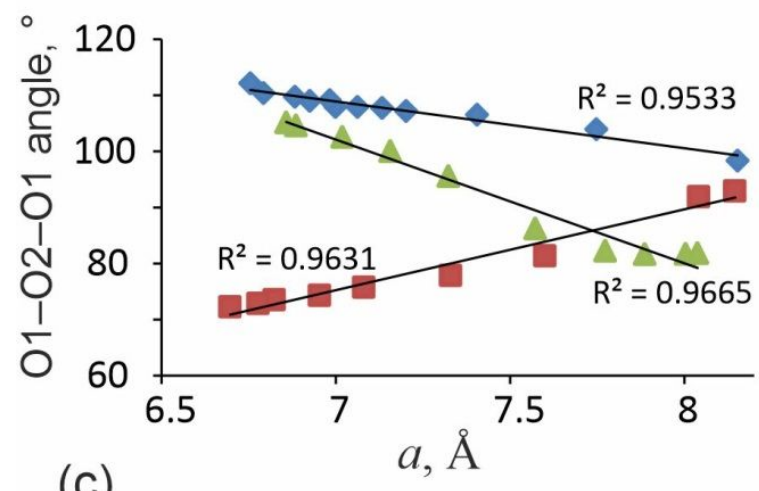

(c)

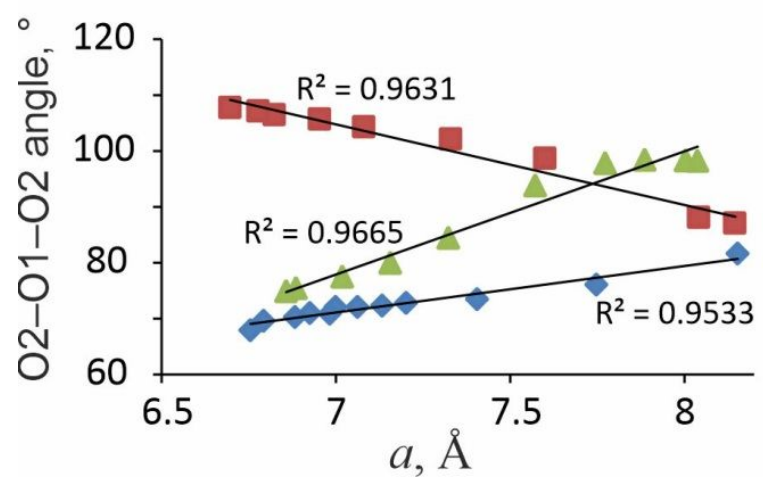

(b)

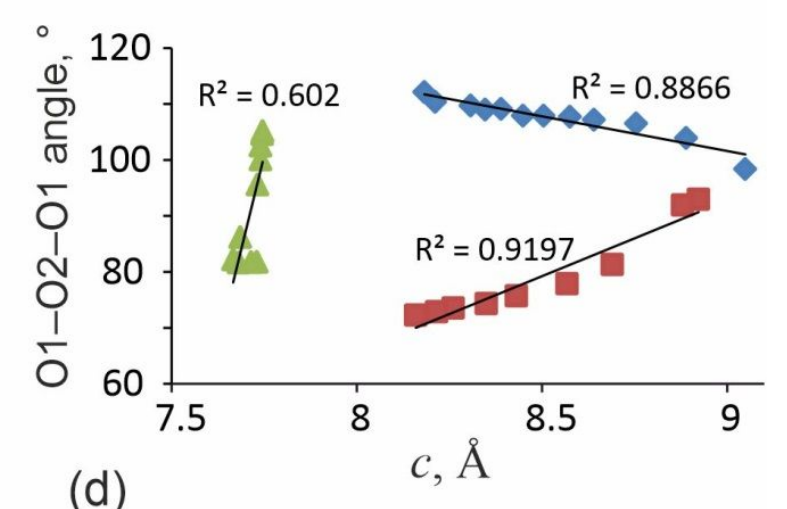

(d)

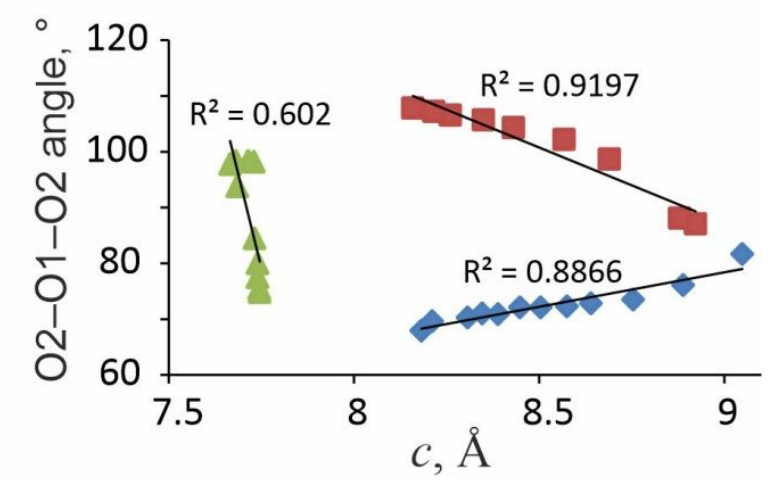

Figure S6. The correlation diagrams between $a$ and $c$ unit-cell parameters and deformations of the $\mathrm{O} 1-\mathrm{O} 2-\mathrm{O} 1(\mathrm{a}, \mathrm{b})$ and $\mathrm{O} 2-\mathrm{O} 1-\mathrm{O} 2(\mathrm{c}, \mathrm{d})$ angles of the four-membered rings for danburite group minerals. 